\title{
The Effect of Atmospheric Plasma on a Protein Thermal Shift Assay of Powdered Whey Protein Isolate
}

\author{
Danielle Aguilar
}

Department of Exercise Science, University of South Florida, United States

daniellea@mail.usf.edu 


\begin{abstract}
:
Whey is both a dietary supplement as well as a food ingredient. It is a byproduct of the cheese making process, and it can be processed further to create three forms, namely whey isolate, whey concentrate and whey hydrolysate. Given whey's complexity and applicability in the food, nutrition and health industries, understanding how changes to its structure contribute to or affect its function is of great interest. One method by which protein structure could be altered is via plasma-surface modification (PSM), an effective surface altering technique of inorganic materials, and there is growing interest in its application on organic materials such as proteins. However, research on the use of PSM to promote structural changes of food proteins has been limited as studies have mostly focused on aqueous solutions and their contributions to pharmacological development. The purpose of this study is to investigate whether the structure of dry powdered whey protein isolate can be modified by atmospheric plasma. The Protein Thermal Shift Assay (PTSA) was implemented to measure structural changes of PSM altered whey protein powder, and a range of protein structure alterations was observed, with a highest total change of $12.77 \%$ between control and treated protein. The applicability of changes in protein structure via atmospheric plasma could have several economical and nutritional benefits as it could be implemented in the process of whey product optimization, which in turn could be of use in the food and nutrition sectors.
\end{abstract}

\title{
Figures:
}

A.

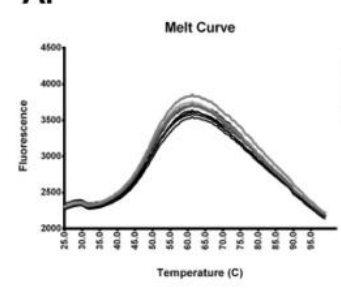

C.

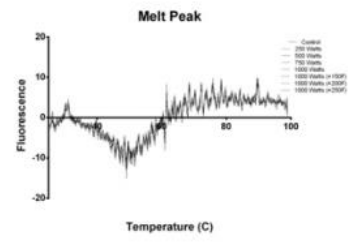

E.

Melt Curve Area Under Curve

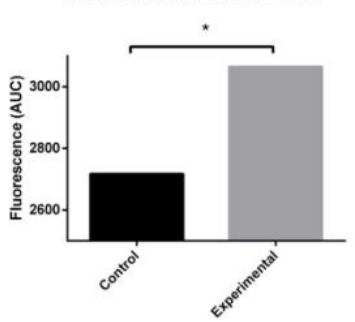

B.

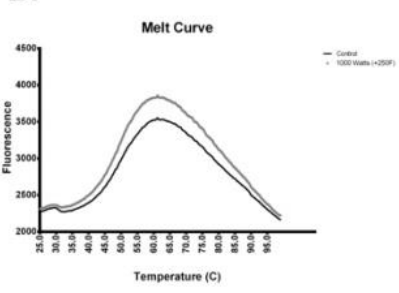

D.

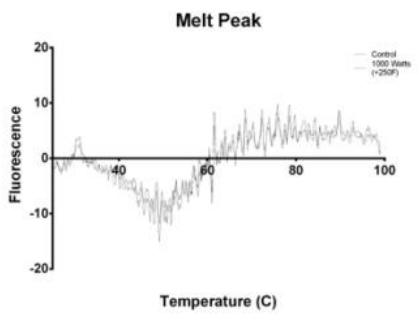




\section{Figure Legend:}

Figure A: Comparison of Melt Curve measurements of 250 watts sample, 500 watts, 750 watts, 1000 watts, 1000 watts (+150F), 1000 watts (+200F) and 1000 watts (+250F) samples as compared to untreated control.

Figure B: Comparison of Melt Curve measurements of 1000 watts (+250F) sample as compared to untreated control.

Figure C: Comparison of Melt Peak measurements of 250 watts sample, 500 watts, 750 watts, 1000 watts, 1000 watts (+150F), 1000 watts (+200F) and 1000 watts (+250F) samples as compared to untreated control.

Figure D: Comparison of Melt Peak measurements of 1000 watts (+250F) sample as compared to untreated control.

Figure E: Comparison of Melt Curve, Area Under Curve of untreated and plasma-treated whey protein powders. Asterisk (*) indicates $12.77 \%$ change in AUC with PSM treatment as compared to untreated control.

\section{Introduction:}

Whey was discovered 3000 years ago, and while it was considered a medicinal agent in the 17th and 18th centuries, the dairy industry viewed whey as a waste product. ${ }^{[1]}$ It was not until the 21 st century when whey processing techniques advanced and whey became one of the most nutritionally available products on the market. ${ }^{[1]}$ Whey proteins have vast applicability, especially in the food and nutrition industries. Therefore, understanding their folding properties and structure is essential in becoming familiar with their functionality. The hydrophobic, electrostatic, and steric parameters of the protein structure all drive the functionality of protein molecules, and therefore conditions that affect or enhance protein stability have the potential to improve both flavor properties of foods as well as therapies and efforts in biopharmaceutical research.

Plasma is the fourth state of matter, existing in the form of ions and electrons. It is a gas that has been charged with electrons in both the negative and positive state. Due to the electrical charges in plasma, the matter is characterized by both high conductivity as well as high internal energy. ${ }^{[2]}$ When plasma ions come in contact with a particular surface, plasma treatment is initiated. Plasma-surface modification (PSM) is a process whereby plasma is applied to the surface of a material in order to alter its chemical properties, such as surface area and hydrophobicity. ${ }^{[2]}$ On an industrial level, PSM is used as a pretreatment to enhance materials such as titanium, silicon, and ceramics, and this is considered to be an economical, reliable, and nontoxic method to achieve a range of chemical modifications to meet a variety of engineering specifications and demands. Briefly, plasma treatment is performed in a vacuum chamber with controllable pressure and temperature. [3] A few processed gasses can be used during plasma treatment, one of which is atmospheric air, in which case a PSM is referred to as atmospheric plasma. ${ }^{4]}$ The ability of atmospheric plasma to directly induce changes in the structure of organic protein powders has not been widely studied yet nor has its effect on the thermal shift assay. 
In this study, PSM-induced protein changes are measured via the Protein Thermal Shift Assay (PTSA). PTSA is a means of screening small molecules in the drug development process with the capability of high-throughput analysis based on very small sample quantities to identify protein structure, stability, ligands, mutations, modifications, and buffer conditions. Briefly, the melting temperature increases yield

a 'thermal shift' that assesses the stabilization of the protein under various conditions. [5] The PTSA has proven to be a popular protein melt analysis method as other protein melt screening processes have so far been inefficient and/or expensive. During the PTSA, the increase in temperature causes proteins to unfold and expose its hydrophobic regions which subsequently bind to the Protein Thermal Shift dye causing it to fluoresce. PTSA has been a popular small molecule screening method in the drug development process due to its high- throughput and accuracy in identifying protein structural changes.

\section{Objective:}

Using a protein thermal shift assay, the objective of this study is to investigate whether the structure of dry powdered whey protein isolate can be modified by atmospheric plasma.

\section{Results \& Discussion:}

This investigation specifically assessed the impact of plasma surface modification on dry powdered whey protein isolate. Protein structure alterations as well as multiple other changes were enacted via Proprietary PSM technology (Ingredient Optimized ${ }^{\text {TM }}$, Plasma Nutrition, USA) to effectively mediate the exposure of organic dry powder materials to plasma. A commercially available dry powder whey protein isolate was used as source material which also served as the control for the experiment. A portion of the whey protein isolate from this batch was separated and exposed to various atmospheric plasma conditions using the PSM technique. Subsequently, PTSA was conducted on both a pristine sample of whey protein isolate (control) and samples of whey protein isolate exposed to atmospheric plasma (experimental) from the same batch.

Effects of PSM on the dry powdered whey isolate were observed via both the Protein Thermal Shift (PTS) melt curves (Figures $A$ and $B$ ) as well as melt peaks (Figures $C$ and $D$ ). An incremental range of protein structural alterations across the tested reaction conditions was observed (Figure $A$ ). The two traces that have the largest difference in fluorescence and thus the largest structural variations are highlighted in Figure B. Those are the traces of the 1000-Watt in a 250F reaction treated protein and untreated control. Similarly, incremental range of protein structural alterations across the tested reaction conditions as measured by the PTS melt peak are shown (Figure C), while Figure D demonstrates a selected protein structural alteration between the two most different traces as measured by the PTS melt peak. All results were significant $(p<0.0001)$. Lastly, a total change was obtained by calculating the area under the curve for a selected melt curve, and an overall $12.77 \%$ difference was observed (Figure E).

Overall, the knowledge of where a protein's stability originates is pivotal in the understanding of that protein's structure and function. [6] The use of atmospheric plasma is promising for application on powdered organic materials. This is particularly true for low pressure atmospheric plasma, as its large uniform area of a well-controlled electron density makes it well suited for the use on dry protein

powder. ${ }^{[2]}$ Furthermore, allowing for entire compound libraries to be screened for stability as well as ligand effects on targets, the PTSA has been a valuable method in the drug discovery industry for over a 
decade. [7] This has been particularly true for therapies that rely on targeted engagement, or a drug's binding affinity to its targeted protein. ${ }^{[8]}$ Here we demonstrate that PSM is an effective method for inducing changes in food protein structure and that PTSA is suitable for detecting those changes. And while PTSA has mostly been used for drug discovery efforts, this analysis has several additional implications for the application of PSM and PTSA, in conjunction, in studies of commercially available protein products, particularly economically in both the food and nutrition industries.

\section{Conclusions:}

PSM offers the ability to selectively alter protein structure with granular control by altering the reaction characteristics of the plasma exposure. This selective control has wide ranging implications not just on the alteration of whey protein but also for alteration of biopharmaceuticals. We conclude that atmospheric plasma is an effective method for inducing changes in protein structure as well as the use of PTSA as an effective means of detecting food protein changes. These findings have several implications in the nutrition and food industries, as it is suggested that the structural changes could improve protein absorption as well as nutritional value when dry protein powder is used in food products.

\section{Limitations:}

This study investigated the application of PSM on only one source of dry powdered whey protein isolate. While the whey protein isolate source material used in this investigation is commonly found in commercial use, there may be variations between the material used and other market- available whey protein isolate powders. Further studies are needed to examine the effect of PSM on a range of dry powder whey protein isolate sources.

\section{Methods:}

A commercially available dry whey protein isolate powder (WPI-90) was used for the study and the specification were verified with a provided certificate of analysis. Within that batch, treated powder was used as source material and untreated as control for the experiment. Source material was exposed to various atmospheric plasma conditions using the Proprietary PSM technology

(Ingredient Optimized ${ }^{\mathrm{TM}}$, Plasma Nutrition, USA) technique described above. The exposure conditions were as follows: a 250, 500, 750 and 1000 Watt plasma at atmospheric (room temperature) reactor temperature as well as 1000 Watt plasma at reactor temperatures of $150 \mathrm{~F}, 200 \mathrm{~F}$ and $250 \mathrm{~F}$. Control powder from the same batch was left untreated.

Protein samples were diluted in phosphate buffer and Applied Biosystems Protein Thermal Shift dye was added. Each sample was run alongside three control samples to ensure accuracy (buffer only, buffer + control protein and buffer + control protein + control ligand). Four replicates of each protein melt reaction sample were tested to ensure statistically significant results. The melt assay was run on a ThermoFisher Quantstudio real-time PCR system. Reactions were individually verified to make sure no pipetting, setup or assignment errors occurred. Melt curve and melt peak data were obtained via Applied Biosystems Protein Thermal Shift software (the melt_peak_data was calculated by taking the first derivative of the melt curve data (melt peak data is equal to $-d(R F U) / d t)$ ). 
Statistical analysis was conducted via Prism 6 (GraphPad Software) using a one-way matched ANOVA $(p<0.05)$.

\section{Funding Statement:}

This work was financially supported by Plasma Nutrition.

\section{Conflict of interest:}

The authors declare no conflicts of interest.

\section{Ethics Statement:}

All experiments were performed in vitro and did not involve the use of any living subject. No fraudulence is committed in performing these experiments or during processing of the data.

\section{References:}

1. Martinez Molina Daniel, Nordlund Pär

The Cellular Thermal Shift Assay: A Novel Biophysical Assay for In Situ Drug Target Engagement and Mechanistic Biomarker Studies. Annual Review of Pharmacology and Toxicology, 56/2016, pages 141-161 DOI:

10.1146/annurev-pharmtox-010715-103715

2.Smithers Geoffrey W.Whey and whey proteins-From 'gutter-to-gold'. International Dairy Journal, 18/2008, pages 695-704. DOI: 10.1016/j.idairyj.2008.03.008

3. Chu P. Plasma-surface modification of biomaterials. Materials Science and Engineering: R: Reports, 36/2002, pages 143-206. DOI: 10.1016/s0927-796x(02)00004-9

4. Jeong $J \mathrm{Y}$,Babayan $\mathrm{S} E$, Tu V J,Selwyn $\mathrm{G} S$ et al. Etching materials with an atmospheric-pressure plasma jet. Plasma Sources Science and Technology, 7/1998, pages 282-285 DOI: 10.1088/0963-0252/7/3/005

5. Shenton M J, Stevens G C. Surface modification of polymer surfaces: atmospheric plasma versus vacuum plasma treatments. Journal of Physics D: Applied Physics, 34/2001, pages 2761-2768 DOI: 10.1088/0022-3727/34/18/308

6. Huynh Kathy, Partch Carrie L. Analysis of Protein Stability and Ligand Interactions by Thermal Shift Assay. Current Protocols in Protein Science, 2015, pages 28.9.1-28.9.14 DOI: 10.1002/0471140864.ps2809s79

7. Becktel Wayne J., Schellman John A. Protein stability curves. Biopolymers, 26/1987, pages 1859-1877 DOI: 10.1002/bip.360261104

8. Jafari Rozbeh, Almqvist Helena, Axelsson Hanna, Molina Daniel Martinez et al. The cellular thermal shift assay for evaluating drug target interactions in cells. Nature Protocols, 9/2014, pages 2100-2122 DOI:

$10.1038 /$ nprot.2014.138 